\title{
Relationships of maternal and paternal birthweights to features of the metabolic syndrome in adult offspring: an inter-generational study in South India
}

\author{
S. R. Veena $\cdot$ S. Geetha $\cdot$ S. D. Leary $\cdot$ J. Saperia $\cdot$ \\ D. J. Fisher • K. Kumaran • P. Coakley • C. E. Stein • \\ C. H. D. Fall
}

Received: 2 June 2006 / Accepted: 6 October 2006 / Published online: 2 December 2006

(C) Springer-Verlag 2006

\begin{abstract}
Aims/hypothesis The association between lower birthweight and metabolic syndrome may result from fetal undernutrition (fetal programming hypothesis) and/or genes causing both low birthweight and insulin resistance (fetal insulin hypothesis). We studied associations between the birthweight of parents and metabolic syndrome in the offspring.

Methods We identified men and women (aged 35-68 years), who had been born in Holdsworth Memorial Hospital, Mysore, India. We also identified the offspring (2046 years) of these men and women. In total, 283 offspring of 193 mothers and 223 offspring of 144 fathers were studied. Investigations included anthropometry, oral glucose tolerance, plasma insulin and lipid concentrations and blood pressure. The metabolic syndrome was defined using WHO criteria.
\end{abstract}

Electronic supplementary material Supplementary material is available in the online version of this article at http://dx.doi.org/ 10.1007/s00125-006-0516-9 and is accessible to authorised users.

S. R. Veena $\cdot$ S. Geetha $\cdot$ K. Kumaran

Holdsworth Memorial Hospital,

Mysore, Karnataka, India

S. D. Leary $\cdot$ J. Saperia $\cdot$ D. J. Fisher $\cdot$ P. Coakley $\cdot$ C. E. Stein $\cdot$

C. H. D. Fall

MRC Epidemiology Resource Centre,

Southampton General Hospital,

Southampton, UK

S. R. Veena $(\square)$

Holdsworth Memorial Hospital,

P.O. Box 38, Mandi Mohalla,

Mysore 570021, India

e-mail: hmhhrcr@sancharnet.in
Results Among the offspring, lower birthweight was associated with an increased risk of glucose intolerance (impaired glucose tolerance, impaired fasting glucose or type 2 diabetes) and higher cholesterol and triacylglycerol concentrations $(p<0.05$ for all adjusted for sex and age). Most outcomes in the offspring, including most individual components of the metabolic syndrome, were unrelated to parental birthweight. However, both maternal and paternal birthweight were inversely related to offspring metabolic syndrome (odds ratio [OR] 0.36 [95\% CI: 0.13-1.01] per $\mathrm{kg}, p=0.053$ for mother-offspring pairs; OR 0.26 [0.07-0.93], $p=0.04$ for father-offspring pairs, adjusted for offspring age, sex, BMI and socioeconomic status). Maternal birthweight was inversely related to offspring systolic blood pressure $(\beta=-2.5 \mathrm{mmHg}[-5.00$ to 0.03$]$ per kg maternal birthweight; $p=0.052$ ).

Conclusions/interpretation Factors in both parents may influence the risk of metabolic syndrome in their offspring. There are several possible explanations, but the findings are consistent with the fetal insulin (genetic) hypothesis.

Keywords Birthweight - Impaired glucose tolerance . India · Insulin resistance - Inter-generational effects . Maternal effects $\cdot$ Metabolic syndrome $\cdot$ Paternal effects

\author{
Abbreviations \\ HMH Holdsworth Memorial Hospital \\ SSTR subscapular:triceps skinfold thickness ratio
}

\section{Introduction}

Lower birthweight is associated with an increased risk of adult CHD and the related disorders insulin resistance, type 2 diabetes and metabolic syndrome [1-3]. The fetal origins 
hypothesis proposes that this reflects undernutrition during critical periods of intrauterine development, which permanently alters (or 'programmes') the body's metabolism [1]. An alternative hypothesis is that genes that either increase insulin resistance or reduce insulin secretion result in impaired insulin-mediated fetal growth and later in CHD and diabetes (fetal insulin hypothesis) [4].

Several recent studies have tested these hypotheses using inter-generational data. These have generally examined disease outcomes in men and/or women in relation to the birthweight of their children. Diabetes in fathers, for example, is associated with lower offspring birthweight [5-9]. The opposite is true for diabetes in mothers, because gestational diabetes causes fetal macrosomia $[10,11]$.
However, lower offspring birthweight is associated with cardiovascular disease and insulin resistance in both parents $[8,12-18]$. Associations between these outcomes in the mother and lower birthweight in her offspring are consistent with the fetal origins hypothesis; a mother who experienced a poor environment during her early development might be programmed both to develop disease and to produce smaller babies (Fig. 1). This hypothesis does not, however, explain the similar associations in fathers. One explanation could be adverse adult behaviours or environmental factors that are shared between fathers and mothers (e.g. smoking or poor diet). These could increase the risk of disease in both parents, and the mothers would be at increased risk of having lower birthweight babies. This is an unlikely
Fig. 1 Inter-generational pathways of disease. The three scenarios are not mutually exclusive. In all cases, associations in mother-offspring pairs may be obscured by maternal gestational diabetes, which causes macrosomia and an increased risk of glucose intolerance in the next generation

\section{Associations between:}

Disease in parents and low birthweight (LBW) in the offspring

(1)

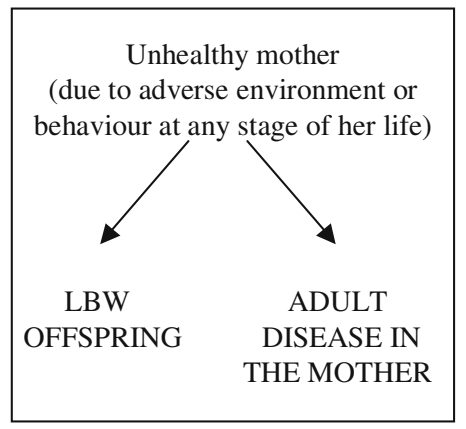

(2)

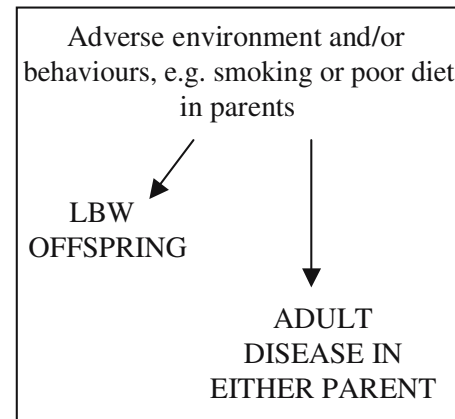

behavioural'

Associations in either parent. These may be attenuated by adjusting for socioeconomic status

Mother-offspring associations only
Low birthweight in parents and disease in the offspring

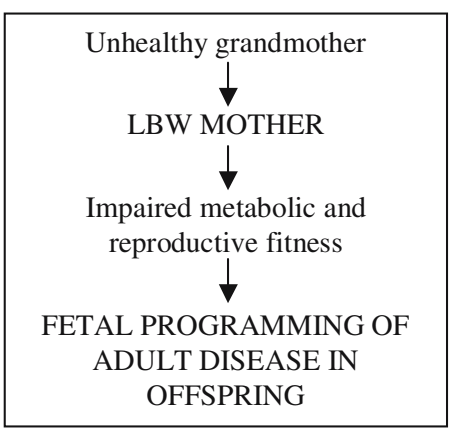

Adverse environment and/or behaviours, e.g. smoking or poor diet Adverse
MOTHER
AND/OR
FATHER

DISEASE IN THE
OFFSPRING

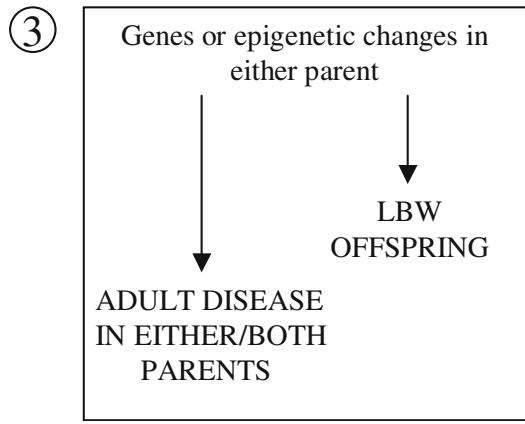

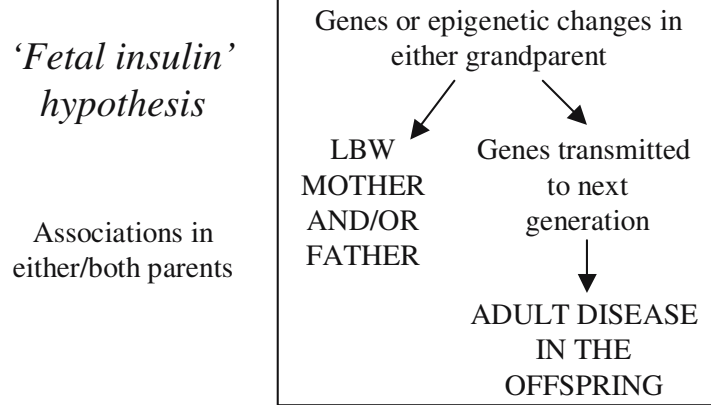


explanation in the above studies, since adjusting for socioeconomic status did not diminish the associations $[12,14-16]$. An alternative explanation is genetic transmission, and the findings from these studies are compatible with the genetic fetal insulin hypothesis. To study this in an Indian population, we identified families living in Mysore, South India, in which two generations were born in one hospital. This enabled us to study the relationship between maternal and paternal birthweight, and insulin resistance, the metabolic syndrome and other CHD risk factors in the young adult offspring. Associations of these outcomes with lower birthweight in both parents or in fathers only would support the fetal insulin hypothesis.

\section{Subjects and methods}

Identification of families The Holdsworth Memorial Hospital (HMH), Mysore, South India, has preserved obstetric records containing the date of birth and birthweight of all babies born in the hospital since 1934. The birth records also contain the names, religion and caste of both parents, the area of the city in which the family lived at the time of birth, and the mother's detailed obstetric history. We used this information to match men and women living in Mysore to their birth records, using strict criteria [19]. Since the baby's name is not recorded in the birth records (Indian babies are named several weeks after birth) and many adults do not know their date of birth or age, the process relies on a match to both parents' names and an exact match of the number, sex and age of a subject's older siblings with their mother's recorded obstetric history.

First generation In 1993, people born in the hospital were identified by a house-to-house census of a 2-square-mile $\left(3.2 \mathrm{~km}^{2}\right)$ area around HMH. Of 7,800 households visited, 1,311 individuals said they were born as singletons in the hospital between 1934 and 1954. Of these, 536 were matched to their birth records and 517 participated in a study to measure the prevalence of CHD [19] and abnormal glucose tolerance [20]. Between 1995 and 2001 the original 2 -square-mile $\left(3.2 \mathrm{~km}^{2}\right)$ area was resurveyed and the census extended to an area of 8 square-miles $\left(12.8 \mathrm{~km}^{2}\right)$. A further 4,748 men and women said to be born in HMH between 1934 and 1966 were identified; 2,892 were matched to their records, and 552 agreed to undergo the same investigations, which took place between 1997 and 2003 (Fig. 2).

Second generation Of 1,069 first generation men and women studied, 221 women and 166 men had adult sons and daughters also born at HMH. From the 221 mothers we identified 206 sons and 209 daughters aged $\geq 20$ years living in or near Mysore, making a total of 415 motheroffspring pairs. From the 166 fathers we identified 152 sons and 144 daughters aged $\geq 20$ years making a total of 296 father-offspring pairs. A total of 283 offspring of 193 mothers and 223 offspring of 144 fathers participated in the study (of whom only 19 had data for both parents), and are included in this analysis. The mother-offspring study was carried out from 1997 to 1999; the father-offspring study, during which more mother-offspring pairs were identified and studied, took place from 2001 to 2003.

Clinical examinations Participants attended hospital for investigations after a 12-h overnight fast. Fasting blood samples were collected for measurement of plasma glucose and insulin, and serum total cholesterol and triacylglycerol concentrations. Excluding those who were known to have type 2 diabetes (20 men and 23 women in the first generation and one man and three women in the second generation), subjects were given a 75-g oral glucose load and further blood samples for plasma glucose and insulin were collected $120 \mathrm{~min}$ later. Fasting insulin values were not available for eight fathers, five mothers and 39 offspring because of difficult venesection, sample haemolysis or technical problems in the laboratory. Blood pressure was measured with an automated recorder (Dinamap 8100; Critikon, Tampa, FL, USA) after the subjects had been lying down for $5 \mathrm{~min}$.

In order to define CHD, a 12-lead ECG was recorded, the Rose chest pain questionnaire was administered [21], and subjects were asked if they had a history of coronary artery bypass surgery. Weight, height, skinfold thicknesses (triceps and subscapular), and hip and waist circumferences were measured by one of three observers. Information on smoking, alcohol consumption and socioeconomic status were obtained by questionnaire. Socioeconomic status was assessed using the Kuppuswamy score [22], a standard questionnaire method for urban Indian populations, based on family size, housing (type of locality, number of people per room, basic amenities), education, occupation and income. The $\mathrm{HMH}$ Ethics Committee granted permission for the study and informed verbal consent was obtained from participants.

Plasma glucose concentrations were analysed using a standard glucose oxidase method and insulin concentrations were measured using an in-house time resolved, dissociation-enhanced lanthanide fluoroimmunoassay method [23]. Assays for the mother-offspring study and father-offspring study were carried out at the Department of Biochemistry, Southampton General Hospital, Southampton, UK, and the Diabetes Research Centre, KEM Hospital, Pune, India, respectively. Serum total cholesterol, HDL-cholesterol and triacylglycerol concentrations were measured by standard enzymatic methods. 


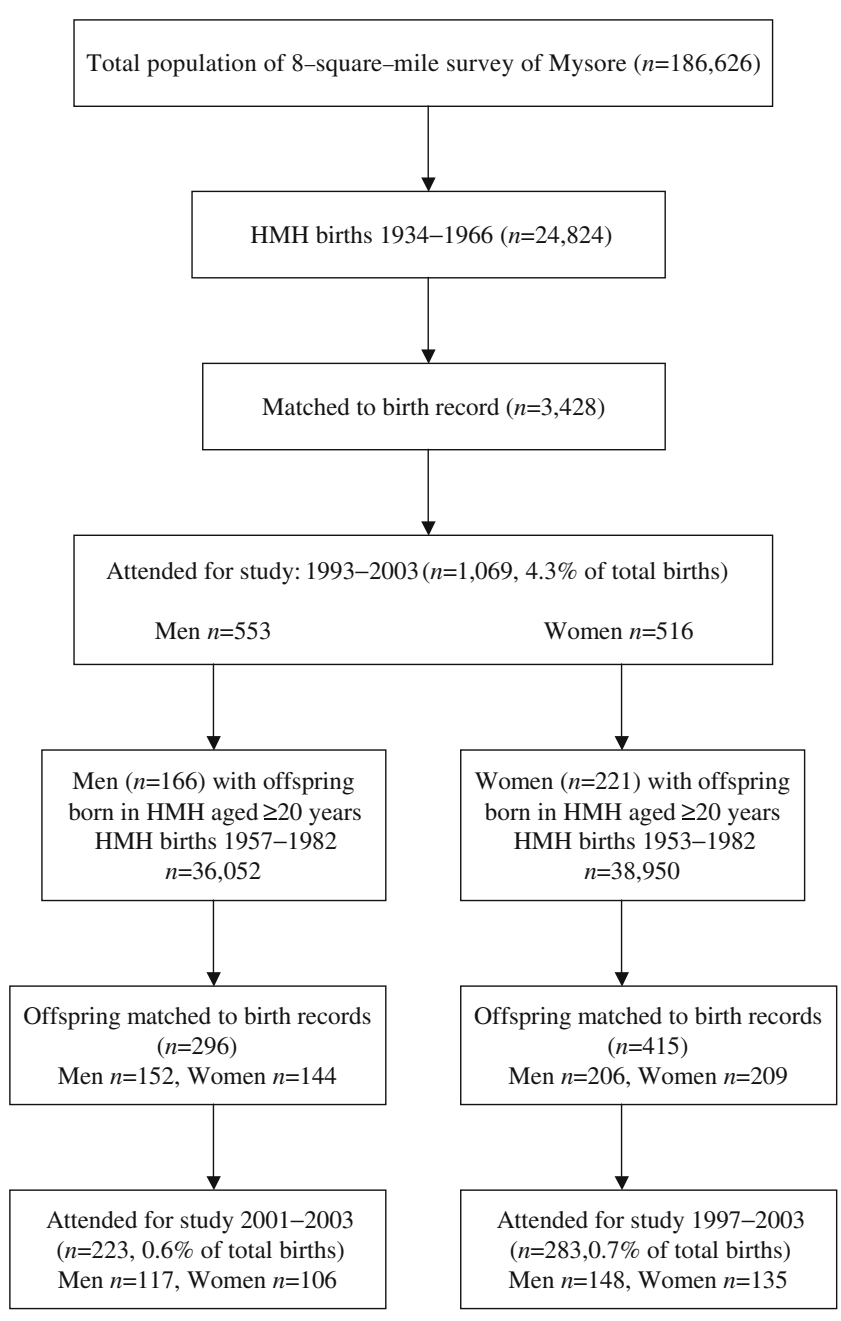

Fig. 2 Flow-chart of study procedures. $H M H$ Holdsworth Memorial Hospital; 8 square miles $=12.8 \mathrm{~km}^{2}$

Type 2 diabetes was defined as either a history of diabetes diagnosed by a doctor and requiring medication, or a fasting glucose concentration of $\geq 7.0 \mathrm{mmol} / \mathrm{l}$, or a 120 min glucose concentration of $\geq 11.1 \mathrm{mmol} / 1$ [24]. IGT was defined as a fasting plasma glucose concentration of $<7.0 \mathrm{mmol} / \mathrm{l}$ and a 120 -min glucose concentration of $\geq 7.8$ and $<11.1 \mathrm{mmol} / \mathrm{l}$. IFG was defined as a fasting glucose concentration of $\geq 6.1$ and $<7.0 \mathrm{mmol} / \mathrm{l}$ and a 120 -min glucose concentration of $<7.8 \mathrm{mmol} / \mathrm{l}$. The presence of IFG, IGT or diabetes was defined as 'glucose intolerance'. Insulin resistance was estimated using the homeostasis model assessment equation [25]. The metabolic syndrome was defined on the basis of WHO criteria [24] as glucose intolerance, and insulin resistance values $\geq$ the 75 th percentile value for our population, together with any two of the following: raised arterial pressure (systolic blood pressure $\geq 140 \mathrm{mmHg}$ or diastolic blood pressure $\geq 90 \mathrm{mmHg}$ ), raised serum triacylglycerol concentration $(\geq 1.7 \mathrm{mmol} / \mathrm{l})$, low serum HDL-cholesterol concentration
( $<0.9 \mathrm{mmol} / \mathrm{l}$ for men; $<1.0 \mathrm{mmol} / \mathrm{l}$ for women) and central obesity (WHR $>0.90$ for men; $>0.85$ for women or BMI $\left.>30 \mathrm{~kg} / \mathrm{m}^{2}\right)$.

ECGs were Minnesota coded [26] by two independent coders, who had no access to the birth data. CHD was defined as the presence of typical angina according to Rose/WHO questionnaire [21], or ECG Minnesota codes 1-1 or 1-2 (Q and Q-S codes) [26] or a history of coronary artery angioplasty or bypass graft surgery.

Statistical methods Statistical analysis was carried out using STATA version 6 (Stata Corporation, College Station, TX, USA). Birth measurements were often recorded in imperial units and were converted into metric values. Distribution was skewed for parent and offspring subscapular:triceps skinfold thickness ratio (SSTR), triacylglycerol and insulin concentrations, and insulin resistance, as well as for offspring BMI and 120-min plasma glucose concentrations. These values were $\log$ transformed to satisfy assumptions of normality. The fathers' fasting and 120-min plasma glucose concentrations and offspring age and fasting glucose concentrations did not transform well using logarithms and these were 'normalised' by ranking each variable and assigning a new value to create normal scores.

Associations between the parent's birth size and outcomes in the offspring were examined by multiple linear and logistic regression, using variables as continuous where appropriate. To overcome the difficulty of mothers and fathers having more than one child in the study, multi-level models were used, allowing us to include the maximum number of parent-offspring pairs. Random effects (including random slopes for continuous outcomes) were estimated using the generalised linear latent and mixed models (GLLAMM) command [27]. Model sensitivity was investigated by comparing the coefficients with those obtained when the number of quadrature points was increased or decreased by four. Sizes of regression effects between offspring of mothers and offspring of fathers were compared by first calculating the standardised beta coefficients, which can also be interpreted as partial correlations; these were converted to $z$-scores using Fisher's transformation, and compared using $z$-tests.

\section{Results}

General characteristics of study cohort Among motheroffspring pairs, $43.8 \%$ were Hindus, $47.7 \%$ were Muslims and $8.5 \%$ were Christians. Corresponding values for fatheroffspring pairs were $33.6,55.2$ and $11.2 \%$. The characteristics of the parents and offspring are shown in Table 1. The parents' birthweights were similar to those of all other births in the hospital during the relevant years (1934 to 
1966; studied $n=337$ vs not studied $n=24,487 ; 2,730$ (SD 413) g vs 2,720 (461) $\mathrm{g} ; p$ for difference $=0.7$ ). The offspring were heavier at birth than the other babies born in the relevant years (1953 to 1982 ; studied $n=487$ vs not studied $n=38,463 ; 2,897$ (455) g vs 2,836 (479) $\mathrm{g} ; p$ for difference $=0.01$ ).

Relationships of offspring birthweight to their own adult outcomes Among all the offspring combined $(n=487)$, 120-min glucose, cholesterol and triacylglycerol concentrations, systolic blood pressure, WHR, SSTR, and the prevalence of IGT, diabetes, glucose intolerance and the metabolic syndrome increased with age $(p<0.05$ for all). All except SSTR rose with increasing BMI ( $p \leq 0.01$ for all). Women were more insulin-resistant than men $(p=0.009)$, while men had higher triacylglycerol concentrations, WHR, SSTR and systolic blood pressure $(p<0.001$ for all). Twenty-four offspring were defined as having CHD; one had a history of bypass surgery, 23 had Rose questionnaire angina, and none had Q-wave ECG changes.

Table 2 shows outcomes in the offspring according to their own birthweight. In the offspring combined, glucose intolerance, total cholesterol and triacylglycerol concentrations, and SSTR were inversely related to birthweight. These associations strengthened after adjusting for BMI, when 120-min glucose, IGT and insulin resistance also became significantly inversely related to birthweight. The trends were similar in offspring of mothers and offspring of fathers. Apparently stronger inverse associations of insulin resistance and cholesterol with birthweight in offspring of fathers than in offspring of mothers (Table 2) were not statistically significantly different. The associations did not change after adjusting for socioeconomic status, and were similar after excluding offspring whose mother was found to have diabetes in our study (we did not have data on diabetes during pregnancy).

Relationships of the parent's birth size to outcomes in the offspring Among the mother-offspring pairs, maternal birthweight was inversely related to the risk of CHD in the offspring (Table 3). After adjusting for offspring BMI and socioeconomic status, inverse associations of borderline statistical significance were seen between maternal birthweight and offspring systolic blood pressure and the metabolic syndrome. These associations remained similar after further adjustment for offspring birthweight (offspring CHD, metabolic syndrome and systolic blood pressure $p<0.05$ for all) and maternal systolic blood pressure (offspring systolic blood pressure $p=0.01$ ).

Among the father-offspring pairs, there were no direct associations between paternal birthweight and offspring outcomes (Table 3). After adjusting for offspring BMI and socioeconomic status, an inverse association was seen between paternal birthweight and offspring metabolic syndrome. This association was similar after further adjusting for offspring birthweight $(p=0.056)$. There were no significant differences in the strength of associations between mother-offspring and father-offspring groups.

Outcomes in the parents We examined risk factors in the parents according to their own birthweight (see Electronic supplementary material [ESM] Table 1). There was an inverse association between birthweight and adult SSTR. After adjusting for adult BMI, we also found an inverse association between birthweight and adult triacylglycerol concentrations.

We also examined outcomes in the parents according to offspring birthweight (see ESM Table 2). The prevalence of diabetes among mothers, and of glucose intolerance among fathers, showed positive associations with offspring birthweight, both findings being of borderline statistical significance. Of 34 cases of CHD among the parents, one had a history of bypass surgery, 12 had Q-wave changes on ECG and 21 had Rose questionnaire angina. The risk of CHD among mothers was inversely related to offspring birthweight (borderline statistical significance). There were no associations between offspring birth size and the metabolic syndrome or insulin resistance in the parents.

\section{Discussion}

The main aim of this inter-generational study was to examine cardiovascular risk factors in Indian adults in relation to their parents' birthweight. There were inverse associations, of borderline statistical significance, and of similar strength, between the birthweight of both mothers and fathers and the risk of metabolic syndrome in their offspring. We also observed inverse associations between maternal birthweight and offspring CHD risk and systolic blood pressure. None of the other outcomes, including most of the individual components of the metabolic syndrome, were related to the birthweight of the parents.

Several mechanisms could have produced inverse relationships between parental birthweight and offspring outcomes (Fig. 1). First, exposure of a female fetus to an adverse intrauterine environment could permanently alter the structure of her reproductive tract, the quality of her ova (the lifetime supply of which develops in utero), her growth potential and/or her metabolism, resulting in impaired fetal development when she becomes pregnant, which in turn programs disease in her offspring. Theoretically, exposure of a male fetus could have long-term effects on sperm quality, leading to abnormalities of fetal development and later disease, but we know of no examples of this. 


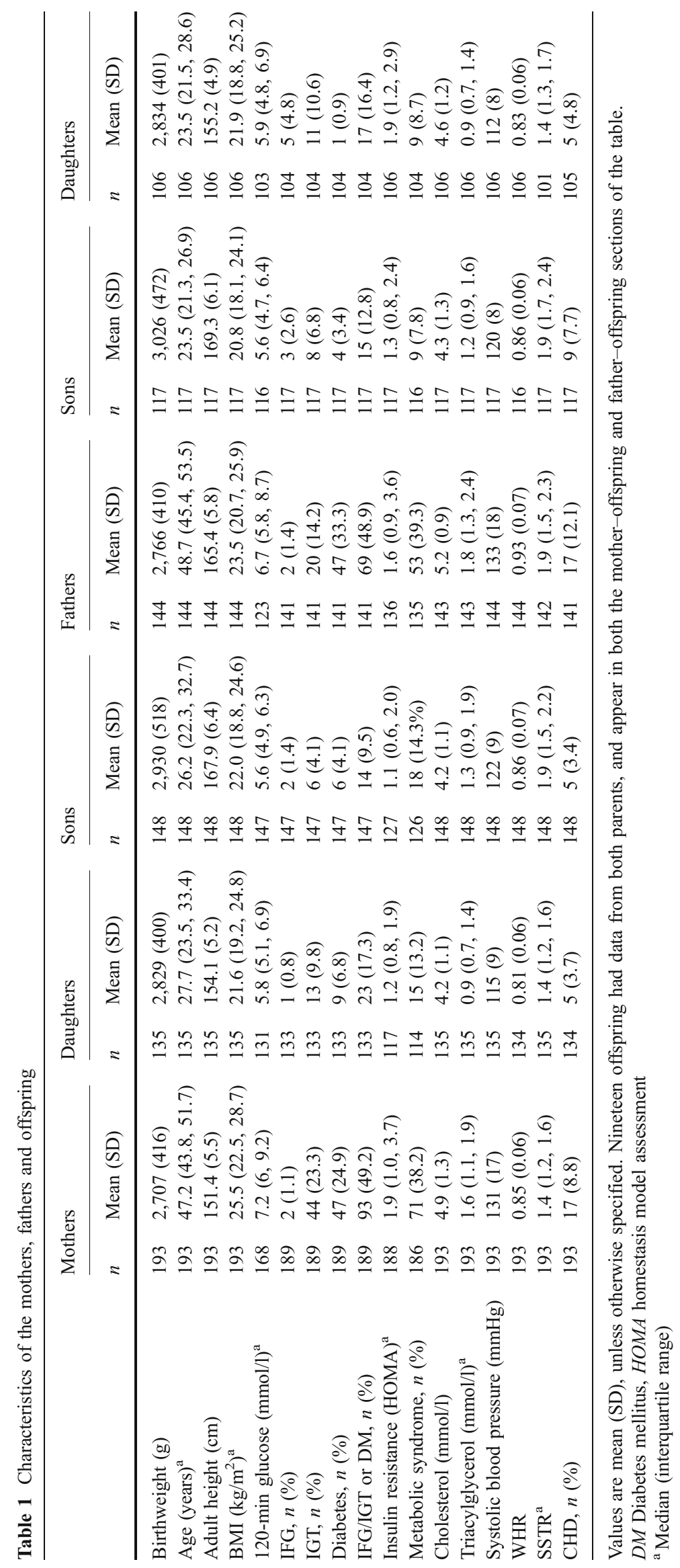


Maternal-fetal programming could explain the inverse associations between maternal birthweight and offspring CHD and blood pressure. Only one other study has examined offspring outcomes according to parental birthweight. This showed, in the UK as in Mysore, an inverse association between maternal birthweight and offspring blood pressure [28]. Second, an adverse adult environment (e.g. socioeconomic deprivation) or behaviours (e.g. smoking or poor diet) that cause both low birthweight and adult disease could be shared by both parents, persist in the same family and result in both mother-offspring and fatheroffspring associations. This explanation seems unlikely in India, where CHD and the metabolic syndrome are predominantly diseases of higher socioeconomic status, although effects of socioeconomic status in childhood may differ from effects in adult life [29]. Smoking is common among Indian men, but very few Indian women smoke. Third, there may be common genes, or epigenetic changes, that result in both low birthweight and adult disease, and could account for both mother-offspring and fatheroffspring associations. Our study cannot distinguish between these three possibilities, but does suggest both paternal and maternal effects.

Studies in western populations $[5-8,12-18]$ and in India $[30,31]$ have examined parental outcomes in relation to offspring birthweight. The western studies showed inverse relationships between offspring birthweight and paternal and maternal insulin resistance measured in middle-to-old age $[8,13]$. The Indian studies had shorter follow-up times than ours, the parents were younger and the offspring were children. Both showed the expected positive associations of maternal insulin resistance and offspring birthweight [30, 31]. Paternal insulin resistance was unrelated to offspring birthweight in one study [30] and positively related in the other [31]. In Mysore parent and offspring birthweights were correlated [32], and if the inverse associations between parental birthweight and offspring metabolic syndrome in our study were genetic in origin, we would expect to see similar inverse associations between offspring birthweight and parental outcomes. The absence of such associations in our study could be explained by the small sample size, effects of medications and disease complications in this older age group, and, in mother-offspring pairs, macrosomic effects of maternal gestational diabetes.

As in other populations [1-3, 10, 11, 33, 34], we found inverse associations between the birthweight of the offspring and their own glucose intolerance and lipid concentrations in later life, adding to evidence that lower birthweight is linked to a higher risk of adult disease. As in most such studies, associations between risk factors and birthweight strengthened after adjustment for adult BMI. BMI adjustment and its interpretation have been controver- sial [35]. Our findings suggest either that at any given adult BMI, the risk factor profile is more adverse in men and women of lower birthweight, or that an increase in BMI between birth and adult life is important for the development of increased CHD risk.

Among the parents, birthweight was also inversely related to SSTR, and (after BMI adjustment) to triacylglycerol concentrations, but not to risk of glucose intolerance or the metabolic syndrome. The lack of an association between birthweight and adult glucose intolerance were reported in an earlier study of the Mysore cohort, of which our parents' sample was a subset [20]. The risk of glucose intolerance in the earlier study was increased in men and women of higher ponderal index at birth [20] and we suggested that this could reflect the effects of gestational diabetes. A possible explanation for the difference in trends with birthweight in the two generations is that effects of gestational diabetes may manifest only in old age. Unlike other populations [36-39], we did not find associations, in either generation, between birthweight and blood pressure. We suggested in the older cohort [40] that this may have been because a large number of subjects were on antihypertensive medication. The absence of an association in the younger cohort, however, suggests that birthweight is simply not related to blood pressure in this population.

A strength of our study was that birth data were extracted from hospital birth records. However, it was limited to people born in one hospital, still living in Mysore, able to provide enough information to match them to their birth records and willing to take part in the study. They comprised a very small proportion of the original births in the hospital $(4.3 \%$ for the first generation and $<1.0 \%$ for the second). Lack of statistical power is therefore a major weakness of the study. Losses to follow-up would have included death (infant mortality rate ranged between 106 and 200 per 1000 live births, and childhood mortality between 162 and 300 in the period 1934 to 1982), migration out of Mysore, and failure to match subjects to their birth records. By using strict criteria, we ensured accurate matching, but with the disadvantage that many likely matches were excluded. Data for the second generation were only available if they were born in $\mathrm{HMH}$. There is a large choice of public and private maternity hospitals in contemporary Mysore. Almost all the first generation subjects had children, but most were born elsewhere; $15 \%$ of Mysore births take place at $\mathrm{HMH}, 3 \%$ at home and the rest at other hospitals. Few of the offspring in our study had birth data from both parents; the ideal study would have compared mother-offspring and fatheroffspring effects in the same subjects. Another weakness of the study was that CHD was defined using the Rose chest pain questionnaire and Q-wave ECG changes, both of which may be non-specific. 


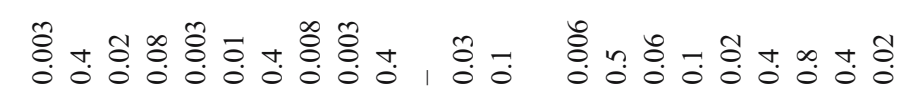

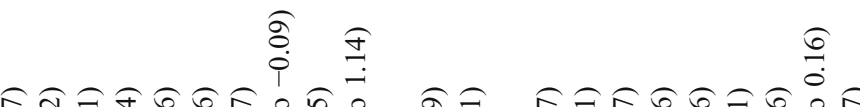

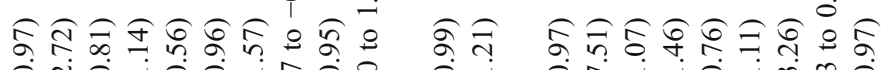

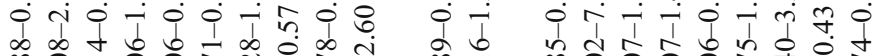

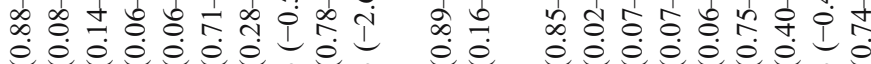

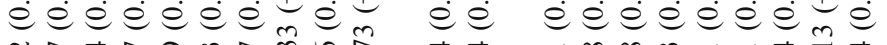

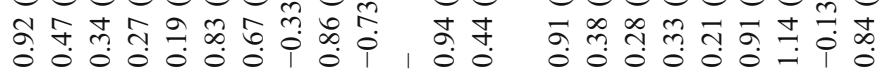

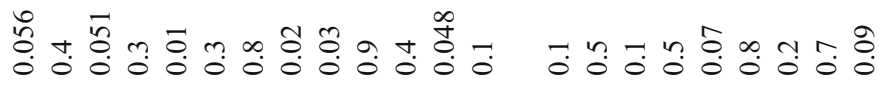

毒

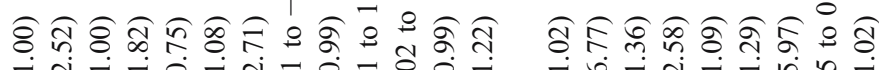
Ti

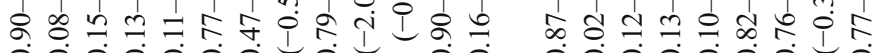

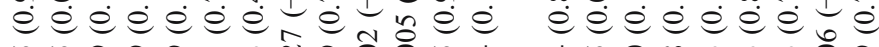

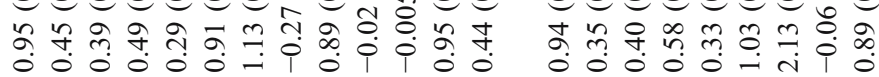

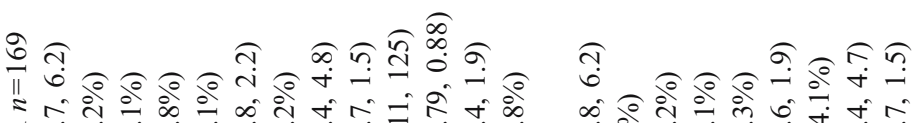

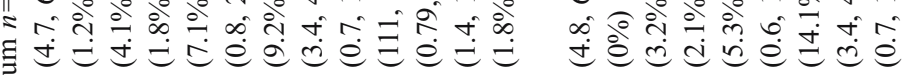
范茫n

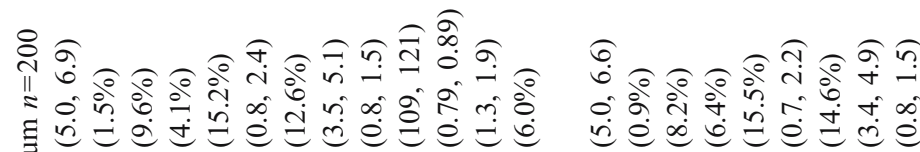
t.

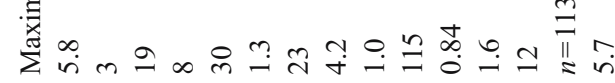

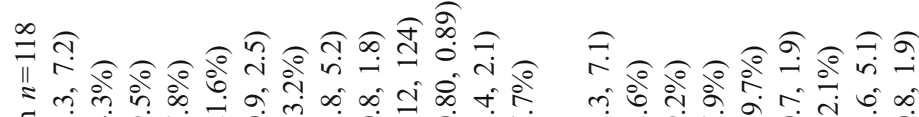

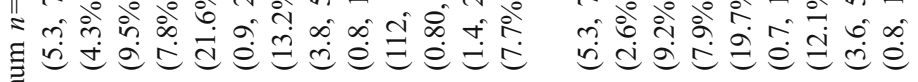
高

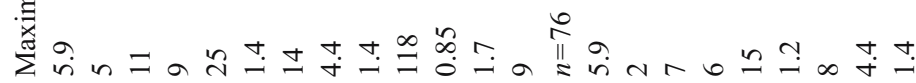

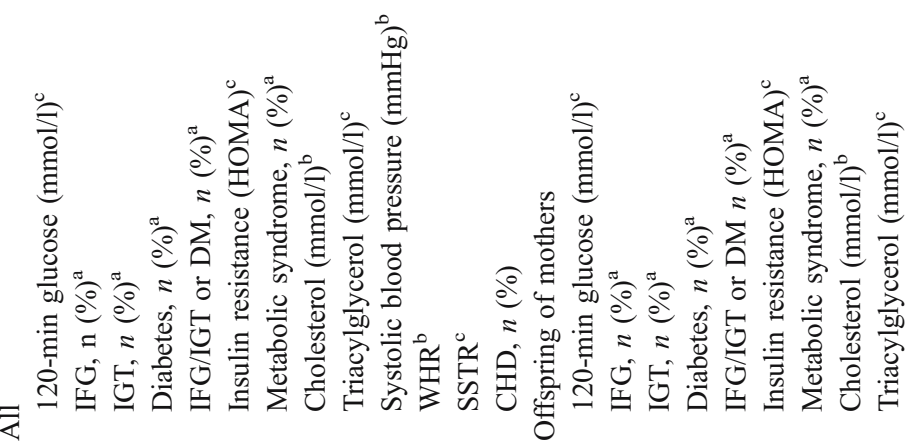




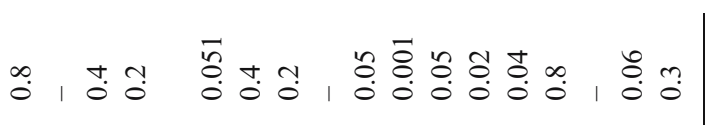

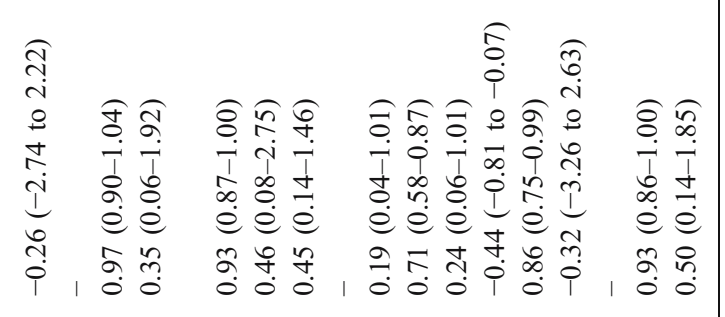

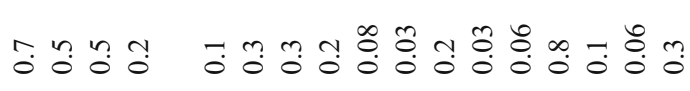

要害

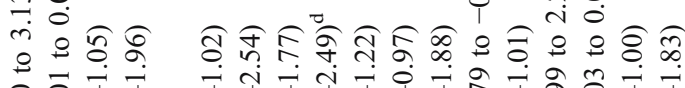

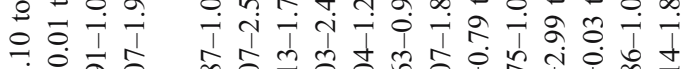

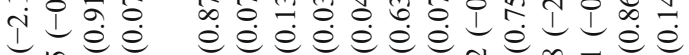

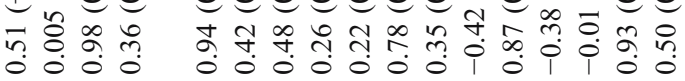

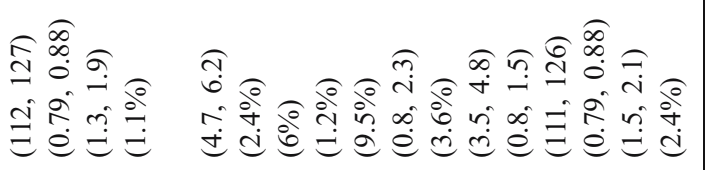

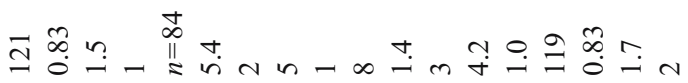

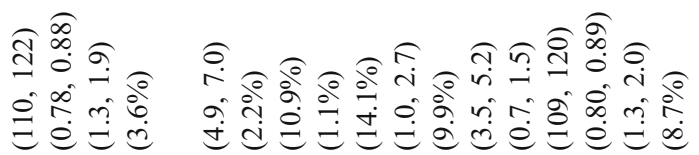

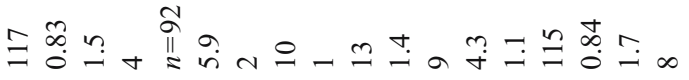

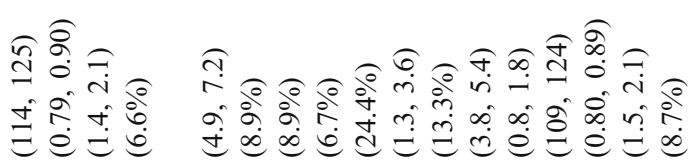

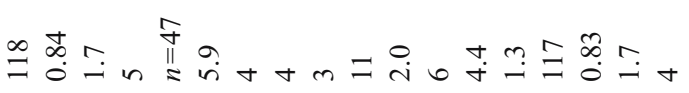

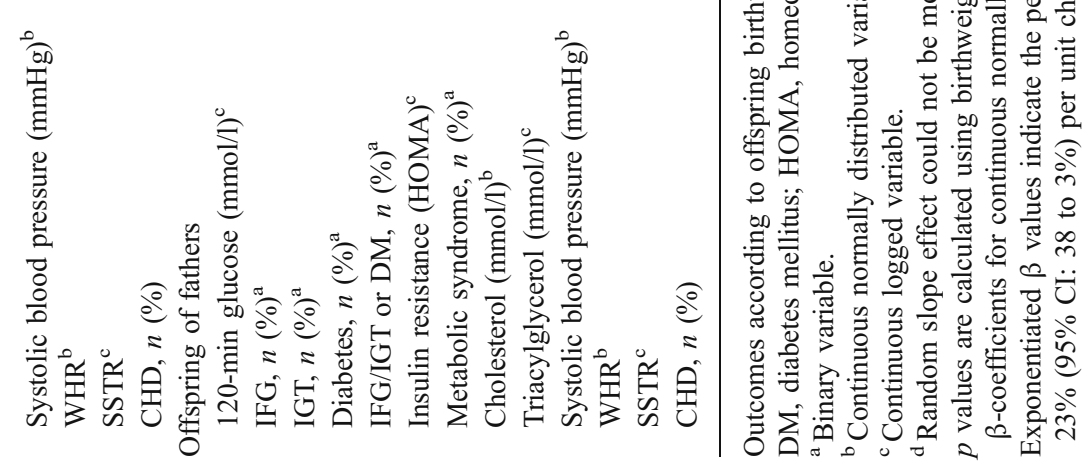




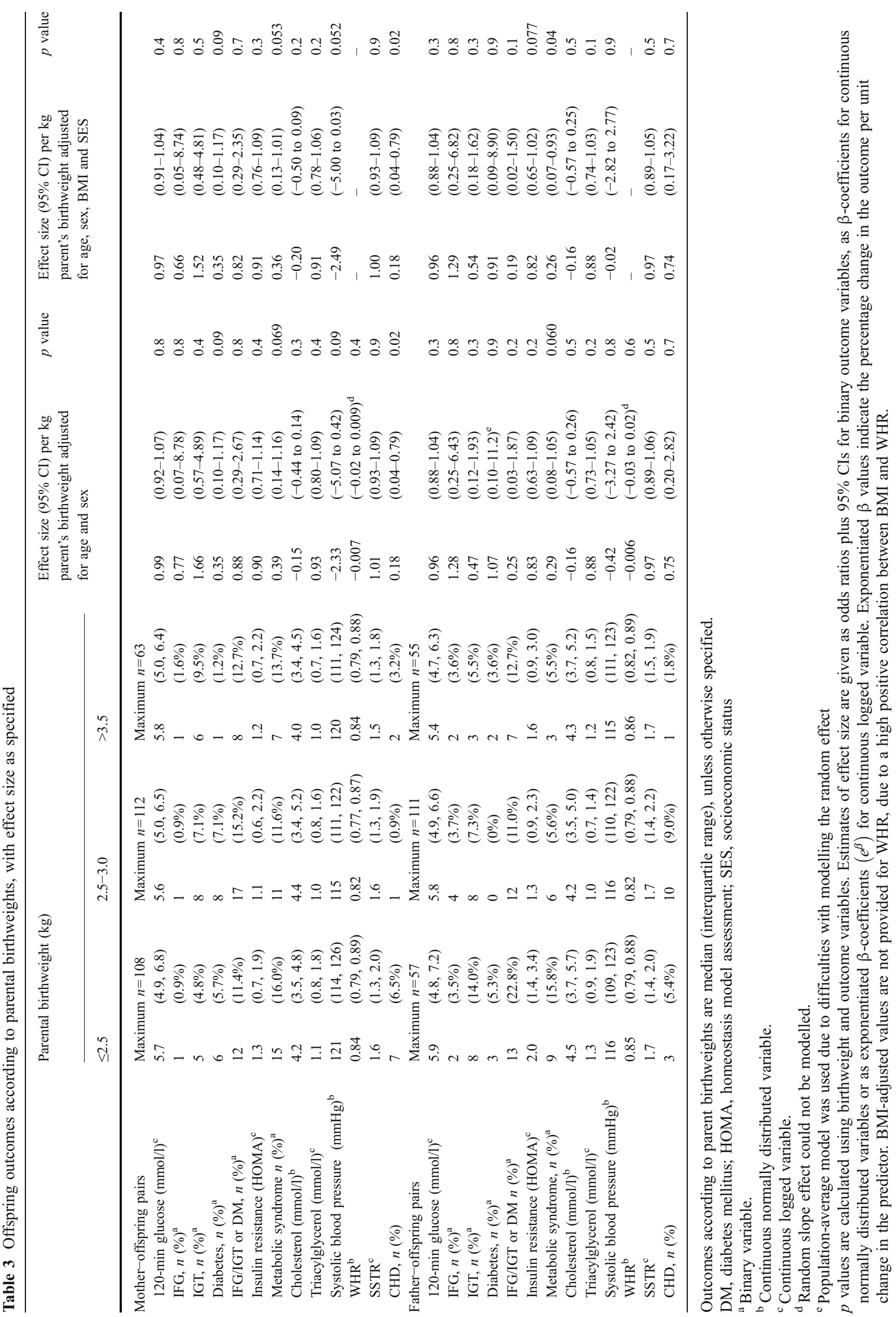


In conclusion, a small sample size was a major weakness of our study. This highlights the difficulty of obtaining good inter-generational data using routine record systems in a developing country. Our study raises more questions than it answers. Most of the outcomes we studied, including the individual components of the metabolic syndrome, were unrelated to parental birthweight. The inverse associations between both maternal and paternal birthweight and metabolic syndrome in the offspring were of borderline significance. These may be chance findings, but are consistent with inter-generational studies (of parental outcomes in relation to offspring birthweight) in western populations. It suggests that factors in both parents may contribute to the risk of metabolic syndrome in the offspring. There are several possible explanations for this, but the findings are most consistent with the fetal insulin (genetic) hypothesis. Further studies in developing countries, using data from cohorts with better follow-up rates are required.

Acknowledgements We are grateful to the men and women who participated and B. D. R. Paul and S. C. Karat, Medical Directors of HMH. We thank N. Venkatachala and the Medical Records staff for access to birth records. M. N. Jayakumar, T. Lawrence, K. J. Chachyamma, M. N. Swarnagowri, M. Surekha, A. Saroja, G. Gopal Singh, T. K. Srinivas and S. Shobha contributed to the study. We thank P. Wood, Endocrine Laboratory, Southampton General Hospital, UK, and C. S. Yajnik and D. S. Bhat, Diabetes Centre, KEM Hospital, Pune, India, for laboratory assays. ECGs were coded by N. Keen and C. Rose. We acknowledge the support of SNEHA-India. The study was funded by the Department for International Development, UK and the Wellcome Trust.

Duality of interest The authors declared that there was no duality of interest regarding this study.

\section{References}

1. Barker DJP (1998) Mothers, babies and health in later life. Churchill Livingstone, London

2. Hales CN, Barker DJP, Clark PMS, Cox LJ, Fall CHD (1991) Fetal and infant growth and impaired glucose tolerance at age 64 years. Br Med J 303:1019-1022

3. Barker DJP, Hales CN, Fall CHD, Osmond C, Phipps K, Clark PMS (1993) Type II (non-insulin-dependent) diabetes mellitus, hypertension and hyperlipidaemia (syndrome $\mathrm{X}$ ): relation to fetal growth. Diabetologia 36:62-67

4. Hattersley AT, Tooke JE (1999) The fetal insulin hypothesis: an alternative explanation of the association of low birthweight with diabetes and vascular disease. Lancet 353:1789-1792

5. Lindsay RS, Dabelea D, Roumain J, Hanson RL, Bennett PH, Knowler WC (2000) Type 2 diabetes and low birthweight. The role of paternal inheritance in the association of low birthweight and diabetes. Diabetes 49:445-449

6. Davey Smith G, Sterne JAC, Tynelius P, Rasmussen F (2001) Birth characteristics of offspring and parental diabetes: evidence for the fetal insulin hypothesis. J Epidemiol Community Health $58: 126-128$
7. Hypponen E, Davey Smith G, Power C (2003) Parental diabetes and birthweight of offspring: intergenerational cohort study. $\mathrm{Br}$ Med J 326:19-20

8. Wannamethee SG, Lawlor DA, Whincup PH, Walker M, Ebrahim S, Davey Smith G (2004) Birthweight of offspring and paternal insulin resistance and paternal diabetes in late adulthood: cross sectional survey. Diabetologia 47:12-18

9. Krishnaveni GV, Hill JC, Leary S et al (2005) Anthropometry, glucose tolerance and insulin concentrations in Indian children: relationships to maternal glucose and insulin concentrations during pregnancy. Diabetes Care 28:2919-2925

10. McCance DR, Petit DJ, Hanson RL, Jacobsson LT, Knowler WC, Bennett PH (1994) Birthweight and non insulin dependant diabetes: thrifty genotype, thrifty phenotype, or surviving small baby genotype? Br Med J 308:942-945

11. Rich-Edwards JW, Colditz GA, Stampfer MJ et al (1999) Birthweight and the risk for type 2 diabetes mellitus in adult women. Ann Intern Med 130:278-284

12. Davey Smith G, Hart C, Ferrell C et al (1997) Birthweight of offspring and mortality in the Renfrew and Paisley study: prospective observational study. Br Med J 315:1189-1193

13. Lawlor DA, Davey Smith G, Ebrahim S (2002) Birthweight of offspring and insulin resistance in late adulthood: cross sectional survey. Br Med J 325:359-362

14. Lawlor DA, Davey Smith G, Whincup P et al (2003) Association between offspring birthweight and atherosclerosis in middle aged men and women: British Regional Heart study. J Epidemiol Community Health 57:462-463

15. Smith GD, Sterne J, Tynelius P, Lawlor DA, Rasmussen F (2005) Birthweight of offspring and subsequent cardiovascular mortality of the parents. Epidemiology 16:563-569

16. Davey Smith G, Harding S, Rosato M (2000) Relation between infant's birthweight and mothers' mortality: prospective observational study. Br Med J 320:839-840

17. Davey Smith G, Whitley E, Gissler M, Hemminki E (2000) Birth dimensions of offspring, premature birth, and the mortality of mothers. Lancet 356:2066-2067

18. Smith GCS, Pell JP, Walsh D (2001) Pregnancy complications and maternal risk of ischaemic heart disease: a retrospective cohort study of 129290 births. Lancet 357:2002-2006

19. Stein C, Fall CHD, Kumaran K, Osmond C, Cox V, Barker DJP (1996) Fetal growth and coronary heart disease in South India. Lancet 348:1269-1273

20. Fall CHD, Stein CE, Kumaran K et al (1998) Size at birth, maternal weight, and type 2 diabetes in South India. Diabet Med 15:220-227

21. Rose GA, Blackburn H, Gillum RF, Prineas RJ (1982) Cardiovascular survey methods, 2nd edn. WHO, Geneva

22. Kuppuswamy B (1962) Manual of socio-economic status scale. Manasayan, Delhi

23. Toivonen E, Hemmila I, Marniemi J, Jorgensen PN, Zeuthen J, Lovgren T (1986) Two-site time-resolved immunofluorometric assay of human insulin. Clin Chem 32:637-640

24. Department of Noncommunicable Disease Surveillance (1999) Definition, Diagnosis and Classification of Diabetes Mellitus and its complications. Report of a WHO consultation-part 1 . World Health Organization, Geneva, Available from http://www. who.int/diabetes/currentpublications/en/, accessed 28 January 2005

25. Matthews DR, Hosker JP, Rudenski AS, Naylor BA, Treacher DF, Turner RC (1985) Homeostasis model assessment: insulin resistance and beta-cell function from fasting glucose and insulin concentrations in man. Diabetologia 28:412-419

26. Prineas RJ, Crow RS, Blackburn H (1982) The Minnesota code manual of electrocardiographic findings: standards and procedures for measurement and classification. John Wright, Boston 
27. Rabe-Hesketh S, Skrondal A (2005) Multilevel and longitudinal modelling using stata. Stata, College Station

28. Barker DJP (2000) Growth in utero and blood pressure levels in the next generation. J Hypertens 18:843-846

29. Lawlor DA, Harro M, Weddekop N et al (2005) Association of socioeconomic position with insulin resistance among children from Denmark, Estonia, and Portugal: cross sectional study. $\mathrm{Br}$ Med J 331:183

30. Yajnik CS, Coyaji KA, Joglekar CV, Kellingray S, Fall C (2001) Paternal insulin resistance and fetal growth: problem for the 'fetal insulin' and the 'fetal origins' hypotheses. Diabetologia 44:11971201

31. Yajnik CS, Joglekar CV, Pandit AN et al (2003) Higher offspring birth weight predicts the metabolic syndrome in mothers but not fathers 8 years after delivery: The Pune children's study. Diabetes 52:2090-2096

32. Veena SR, Kumaran K, Swarnagowri et al (2004) Intergenerational effects on size at birth in South India. Paediatr Perinat Epidemiol 18:361-370

33. Law CM, Gordon GS, Sheill AW, Barker DJ, Hales CN (1995) Thinness at birth and glucose tolerance in seven-year-old children. Diabet Med 12:24-29
34. Bavdekar A, Yajnik CS, Fall CH et al (1999) Insulin resistance syndrome in 8 -year old children: small at birth, big at 8 years, or both? Diabetes 48:2422-2429

35. Lucas A, Fewtrell MS, Cole TJ (2001) Fetal origins of adult disease - the hypothesis revisited. Br Med J 323:572-573

36. Barker DJ, Bull AR, Osmond C, Simmonds SJ (1990) Fetal and placental size and risk of hypertension in adult life. Br Med J 301:259-262

37. Martyn CN, Barker DJP, Jespersen S et al (1995) Growth in utero, adult blood pressure and arterial compliance. Br Heart J 73:116121

38. Law CM, Sheill AW (1996) Is blood pressure inversely related to birthweight? The strength of evidence from a systemic review of the literature. J Hypertens 14:935-941

39. Huxley RR, Sheill AW, Law CM (2000) The role of size at birth and postnatal catch-up growth in determining systolic blood pressure: a systematic review of the literature. J Hypertens $18: 815-831$

40. Kumaran K, Fall CHD, Martyn CN, Vijayakumar M, Stein C, Shier R (2000) Blood pressure, arterial compliance, and left ventricular mass: no relation to small size at birth in South Indian adults. Heart 83:272-277 